\title{
Autophagy-mediated adaptation of hepatocellular carcinoma cells to hypoxia-mimicking conditions constitutes an attractive therapeutic target
}

\author{
SATOSHI OWADA ${ }^{1}$, HITOSHI ENDO ${ }^{1}$, YUKARI SHIDA ${ }^{1}$, CHISA OKADA $^{2}$, \\ KANAKO ITO $^{1}$, TAKAHIRO NEZU ${ }^{1}$ and MASAYUKI TATEMICHI ${ }^{1}$ \\ ${ }^{1}$ Center for Molecular Prevention and Environmental Medicine, Department of Preventive Medicine, \\ Tokai University School of Medicine; ${ }^{2}$ Support Center for Medical Research and Education, \\ Tokai University, Isehara, Kanagawa 259-1193, Japan
}

Received July 28, 2017; Accepted January 31, 2018

DOI: $10.3892 /$ or.2018.6279

\begin{abstract}
Hepatocellular carcinoma has extremely poor prognosis. In cancerous liver tissues, aberrant proliferation of cancer cells leads to the creation of an area where an immature vascular network is formed. Since oxygen is supplied to cancer tissues through the bloodstream, a part of the tumor is exposed to hypoxic conditions. As hypoxia is known to severely reduce the effectiveness of existing anticancer agents, novel valid therapeutic targets must be identified for the treatment of hepatocellular carcinoma. Generally, autophagy has been reported to play an important role in the adaptation of cancer cells to hypoxia. However, the exact role and significance of this process vary depending on the cancer type, requiring detailed analysis in individual primary tumors and cell lines. In the present study, we examined autophagy induced by cobalt chloride, a hypoxia-mimicking agent, in hepatocellular carcinoma cells with the aim to evaluate the validity of this process as a potential therapeutic target. We observed that treatment with cobalt chloride induced autophagy, including the intracellular quality control mechanism, in an AMPK-dependent manner. Furthermore, treatment with autophagy inhibitors (bafilomycin and LY294002) resulted in significant, highly-selective cytotoxicity and apoptosis activation under hypoxia-mimicking conditions. The knockdown of AMPK also revealed significant cytotoxicity in hypoxia-mimicking conditions. These results clearly demonstrated that autophagy, especially mitophagy, was induced by the AMPK pathway when hepatocellular carcinoma cells were subjected to hypoxic conditions and
\end{abstract}

Correspondence to: Professor Masayuki Tatemichi, Center for Molecular Prevention and Environmental Medicine, Department of Preventive Medicine, Tokai University School of Medicine, 143 Shimokasuya, Isehara, Kanagawa 259-1193, Japan

E-mail: tatemichi@tokai-u.jp

Key words: hypoxia, AMPK, autophagy, apoptosis, hepatocellular carcinoma played an important role in the adaptation of these cells to such conditions. Thus, autophagy may constitute an attractive therapeutic target for the treatment of hepatocellular carcinoma.

\section{Introduction}

Hepatocellular carcinoma is known to be highly malignant and has a poor prognosis, constituting the most common cause of cancer-related deaths worldwide after lung cancer (1). Standard therapies for liver cancer treatment include the administration of gemcitabine and sorafenib (2). However, these have high rates of unfavorable treatment outcomes (3).

Almost all tumors, including hepatocellular carcinomas, contain an heterogeneous vascular network due to angiogenic imbalance and the aberrant proliferation of cancer cells (4-7). Since oxygen is supplied through the bloodstream, anoxic $\left(<0.5 \% \mathrm{O}_{2}\right)$, hypoxic $\left(0.5-1.5 \% \mathrm{O}_{2}\right)$ and normoxic $\left(>1.5 \% \mathrm{O}_{2}\right)$ regions are supposed to intermingle in tumor tissues (8-10). Many studies have reported that hypoxic environments dramatically reduce the effectiveness of existing anticancer agents (11-14). Therefore, there is an urgent need for the discovery of novel targets that would lead to the development of pharmaceutical agents able to exert their anticancer activity in a hypoxic environment.

Induction of the stabilization of hypoxia-inducible factor 1 (HIF-1) is crucial for the adaptation of cancer cells to hypoxic conditions (15), as the stabilized protein is a transcription factor that regulates the expression of various genes in order to shift energy production from oxidative phosphorylation in mitochondria to glycolysis and/or to facilitate angiogenesis (16). It was recently reported that HIF-1-dependent activation of autophagy occurs under hypoxic conditions (17). Autophagy is an intracellular cleaning mechanism in which cellular components are digested by lysosomal enzymes after being isolated from the cytoplasm by a bilayer membrane. Autophagy is induced not only by hypoxia, but also by other types of intense cellular stress including nutrient deprivation, growth factor withdrawal and treatment with anticancer agents (18-20). Hypoxia constitutes a severe metabolic stress and the induction of autophagy is likely to contribute to 
adaptation by allowing damaged organelles and proteins to be properly processed, which is a crucial part of the intracellular quality control system.

However, the significance and roles of autophagy under hypoxic conditions are subject of extreme controversy, as they appear to vary among cancers and/or cell types. Some studies reveal that autophagy contributes to cell survival, whereas others indicate that it negatively affects this outcome (21-23). For that reason, it is necessary to thoroughly analyze the significance of autophagy under hypoxic conditions in individual primary tumors. The present study aimed to clarify the influence of autophagy induced by cobalt chloride, a hypoxia-mimicking agent, on the survival of hepatocellular carcinoma cells, as well as the potential usefulness of this process as a therapeutic target. The results of the present study revealed that autophagy under such conditions was dependent on the AMPK pathway and suppressed apoptosis, whereas blockage of this pathway may constitute an attractive therapeutic target as it reduces the ability of cancer cells to adapt to hypoxia.

\section{Materials and methods}

Reagents. The materials for the present study were obtained from the following sources: LY294002 was obtained from Calbiochem (Merck KGaA, Darmstadt, Germany). Dulbecco's modified Eagle's medium (DMEM), dimethyl sulfoxide, cobalt chloride, bafilomycin A1, MitoTEMPO and a proteinase inhibitor cocktail were purchased from Sigma-Aldrich (St. Louis, MO, USA). MitoTracker Green FM and MitoSOX Red were obtained from Thermo Fisher Scientific (Waltham, MA, USA). Anti-LC3 antibody (1:1,000; rabbit polyclonal; cat no. PM036) was purchased from MBL (Medical and Biological Laboratories Co., Ltd., Nagoya, Japan). The antibodies against phospho-p70 S6 kinase (Thr389) (1:1,000; rabbit monoclonal; cat. no. 9234), p70 S6 kinase (1:1,000; rabbit polyclonal; cat. no. 9202), phospho-Akt (Ser473) (1:1,000; rabbit monoclonal; cat. no. 4058), AKT (1:1,000; rabbit polyclonal; cat. no. 9272), phospho-AMPK $\alpha$ (Thr172) $(1: 1,000$; rabbit monoclonal; cat. no. 2535) and AMPK $\alpha$ (1:1,000; rabbit polyclonal; cat. no. 2532), as well as Alexa Flour 555-(1:1,000; cat. no. 4409) or Alexa Flour 488-conjugated secondary antibodies (1:1,000; cat. no. 4412) were obtained from Cell Signaling Technology (Danvers, MA, USA). Anti-HIF-1 $\alpha$ antibody (1:1,000; rabbit polyclonal; cat. no. GTX127309) was purchased from GeneTex (Irvine, CA, USA), anti-actin antibody (1:10,000; mouse monoclonal; cat. no. 013-24553) was obtained from Wako Pure Chemical Industries (Osaka, Japan). The anti-LAMP1 (1:500; mouse monoclonal; cat. no. sc-20011) and anti-Tom20 (1:500; mouse monoclonal; cat. no. sc-17764) antibodies were obtained from Santa Cruz Biotechnology (Dallas, TX, USA).

Cell lines and culture conditions. The human hepatocellular carcinoma cell lines Huh7 and HepG2 were purchased from the Japanese Collection of Research Bioresources Cell Bank (JCRB Cell Bank, Osaka, Japan). Both cell lines were cultured in DMEM supplemented with $10 \%$ fetal bovine serum (FBS), $50 \mathrm{U} / \mathrm{ml}$ penicillin, $50 \mu \mathrm{g} / \mathrm{ml}$ streptomycin and non-essential amino acids (Gibco BRL; Thermo Fisher Scientific, Paisley, UK). The cells were cultured at $37^{\circ} \mathrm{C}$, under $5 \% \mathrm{CO}_{2}-95 \%$ air.
Cytotoxicity assay. Cytotoxicity assays were performed using the Cell Counting Kit-8 (CCK-8; Dojindo Molecular Technologies, Kumamoto, Japan). In brief, HepG2 or Huh7 cells were seeded in 96 -well plates $\left(1 \times 10^{4}\right.$ cells/well) and incubated for $24 \mathrm{~h}$. The medium was then changed to DMEM with or without $300 \mu \mathrm{M}$ cobalt chloride, followed by addition of serial dilutions of autophagy inhibitors. After another $24 \mathrm{~h}$, the cells were washed with phosphate-buffered saline (PBS) and $100 \mu \mathrm{l}$ of DMEM containing 10\% WST-8 solution was added to each well and each plate was incubated for another $3 \mathrm{~h}$. Subsequently, the absorbance at $460 \mathrm{~nm}$ was assessed and cell viability values were expressed as percentages, with the cell numbers in the corresponding control cultures (absence of autophagy inhibitors under each culture condition) set as $100 \%$.

Western blot analysis. Protein extraction and western blot analysis were performed as previously described (24). The antibody dilutions were used according to the manufacturer's instructions.

Immunostaining analysis. Cells grown on 4-well glass chamber slides (Sigma-Aldrich) were treated with $300 \mu \mathrm{M}$ cobalt chloride $\left(\mathrm{CoCl}_{2}\right)$ for $24 \mathrm{~h}$. After incubation, the slides were washed with PBS, fixed with PBS containing $4 \%$ formaldehyde and $0.1 \%$ Triton X-100 for $30 \mathrm{~min}$ at $25^{\circ} \mathrm{C}$, blocked with $3 \%$ bovine serum albumin in PBS for $1 \mathrm{~h}$ and incubated first with the indicated primary antibody for $1 \mathrm{~h}$ at $25^{\circ} \mathrm{C}$ and then, with the corresponding secondary antibody at $25^{\circ} \mathrm{C}$ for another $1 \mathrm{~h}$. DNA was counterstained with SlowFade mounting medium containing DAPI (4',6-diamidino-2-phenylindole dihydrochloride) that was obtained from Cell Signaling Technology. Images of optical sections with $0.7 \mu \mathrm{m}$ thickness were captured using a Zeiss LSM 700 confocal laser scanning microscope (Carl Zeiss Microscopy, GmbH, Oberkochen, Germany) under a $63 x$ lens objective (numerical aperture, $1.2 \mathrm{~W}$ ). The Alexa Fluor 488 dye was excited by a $488-\mathrm{nm}$ laser and the resulting fluorescence emission was detected through a filter that transmitted wavelengths ranging from 420 to $550 \mathrm{~nm}$. The Alexa Fluor 555 dye was excited by a $555-\mathrm{nm}$ laser and the resulting fluorescence emission was detected through a filter that transmitted wavelengths over $560 \mathrm{~nm}$. The number of puncta was counted using ImageJ v1.51n software (NIH, Bethesda, MD, USA). DAPI was used for nuclear counterstaining and its 405-nm-excited fluorescence emission was detected through a filter that transmitted wavelengths over $420 \mathrm{~nm}$.

Plasmids and stable transfection. The HepG2 cells were transfected with MISSION $^{\circledR}$ short hairpin targeting human AMPKa.1 (sh-AMPK CCGGCCATCCTGAAA GAGTACCATTCTCGAGAATGGTACTCTTTCAGGATG GTTTTT)-containing plasmids (Sigma-Aldrich) using Lipofectamine LTX Reagent with PLUS Reagent (Thermo Fisher Scientific) for $48 \mathrm{~h}$. The cells were then transferred into medium containing $1.5 \mu \mathrm{g} / \mathrm{ml}$ puromycin (Sigma-Aldrich) for 3 weeks for single-cell clone selection to obtain a stable-expression cell line.

Statistical analysis. Bars or symbols in the graphs represent the means \pm standard deviation (SD) generated from at least 
$\mathbf{A}$
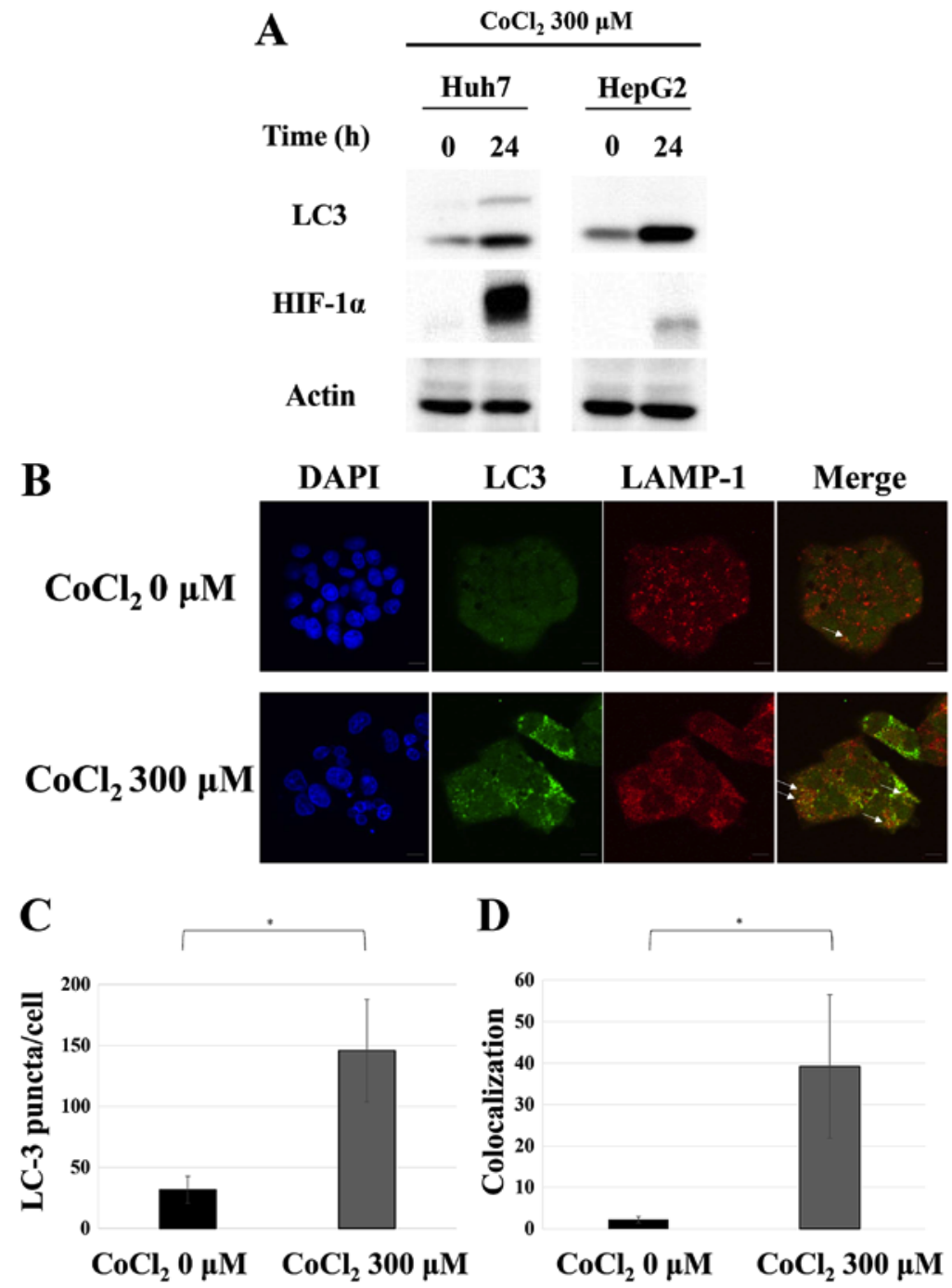

Figure 1. Treatment with cobalt chloride induces the progressive activation of autophagy in hepatocellular carcinoma cells. (A) Huh7 and HepG2 cells were treated with $300 \mu \mathrm{M}$ cobalt chloride $\left(\mathrm{CoCl}_{2}\right)$ for $24 \mathrm{~h}$ and the expression levels of LC3, HIF-1 $\alpha$ and actin were determined by western blot analysis. (B) HepG2 cells were immunostained with anti-LC3 (green) and anti-LAMP-1 (lysosome marker, red). Insets indicate magnified images of the boxed area. Bar, $10 \mu \mathrm{m}$. Arrows indicate colocalization between LC3 and LAMP-1. (C) Punctate dots of LC3 in each cell were counted ( $\left.{ }^{*}<0.05\right)$ and (D) colocalization between LC3 and lysosomes in each cell was assessed using Image $\mathrm{J}$ software $\left({ }^{*} \mathrm{P}<0.05\right)$.

three independent experiments. Significant differences were determined by one-way analysis of variance (ANOVA), two-way ANOVA or t-tests. A P-value of $<0.05$ was considered to indicate a statistically significant difference.

\section{Results}

Autophagy is induced by cobalt chloride treatment. In order to confirm the induction of autophagy in a hypoxic environment, we treated the Huh7 and HepG2 cells with a hypoxia-mimicking agent, cobalt chloride $(25,26)$. This resulted in a significant accumulation of HIF-1 $\alpha$, which is a hypoxia marker, as well as an increase in the band of LC3-II, which is a modified form of LC3 that can be found in autophagosomes and autolysosomes (Fig. 1A). Both changes indicated the induction of autophagy under hypoxia-mimicking conditions. HepG2 cells were also subjected to double immunostaining for LC3 and LAMP1, a lysosomal marker. As displayed in Fig. 1B-D, cobalt chloride treatment resulted in an increase in LC3 puncta, confirming autophagy induction. Furthermore, the observed colocalization of LC3 and LAMP1 indicated the fusion of autophagosomes and lysosomes. These results strongly indicated that autophagy was induced in hepatocellular carcinoma cells under hypoxic conditions

Autophagy induced by cobalt chloride is dependent on the AMPK/mTOR pathway. Subsequently, we investigated in detail the signaling pathway mediating the induction of autophagy by cobalt chloride treatment. The activity of mTOR, a regulator of autophagy, was examined using the phosphorylation status of p70S6K, which is a direct target of mTOR (27), as an indicator. Cobalt chloride treatment led to a significant attenuation of p70S6K phosphorylation, strongly indicative of lower mTOR activity in both cell lines (Fig. 2A). Previous studies have revealed that the phosphorylation of mTOR is positively regulated by AKT (28) and negatively regulated by AMPK (29). Thus, the lower mTOR activity that we observed, may be attributed to a reduction of the AKT activity (e.g., through decreased phosphorylation) and/or an increase in the AMPK activity (e.g., through increased 
A

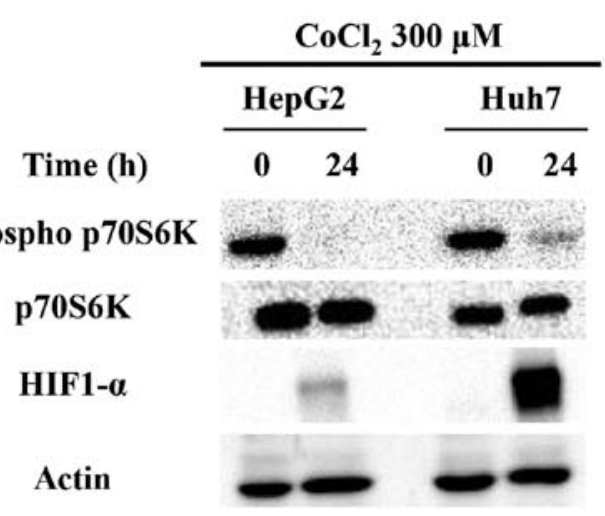

B

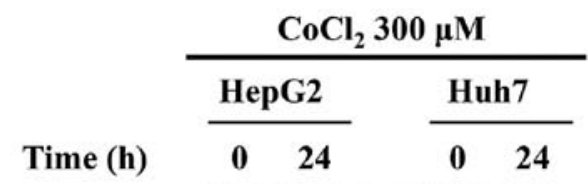

Phospho AMPK

AMPK

Phospho AKT

AKT

Figure 2. Autophagy induced by cobalt chloride is dependent on the AMPK/mTOR pathway. Huh7 and HepG2 cells were treated with $300 \mu$ M cobalt chloride $\left(\mathrm{CoCl}_{2}\right)$ and harvested at the indicated incubation times. (A) Western blot analysis was used to determine the expression levels of phospho-P70S6K, P70S6K, HIF-1 $\alpha$ and actin, as well as (B) those of phospho-AKT, AKT, phospho-AMPK and AMPK.

A

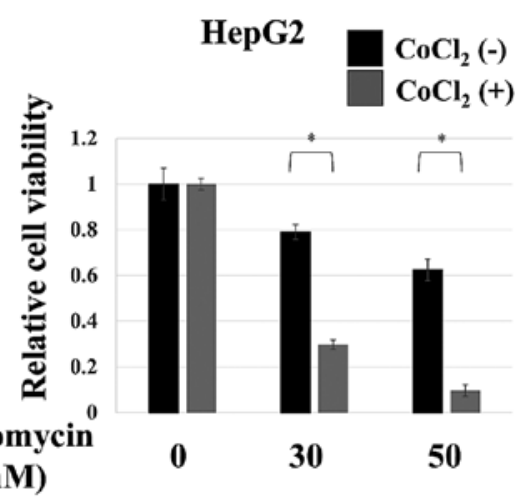

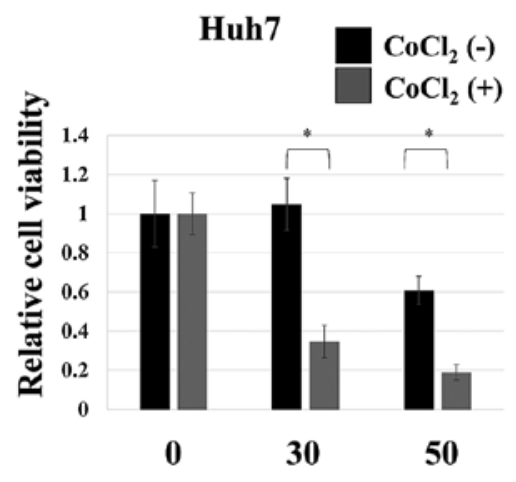
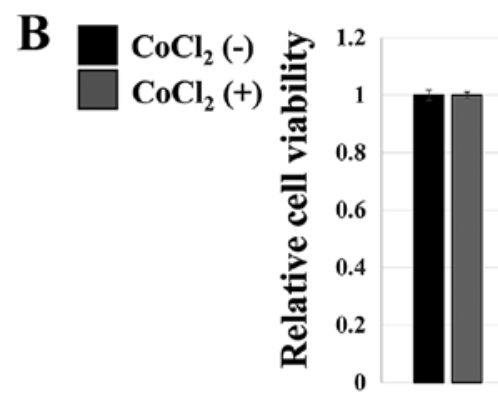

LY294002 ( $\mu$ M)

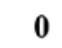

20

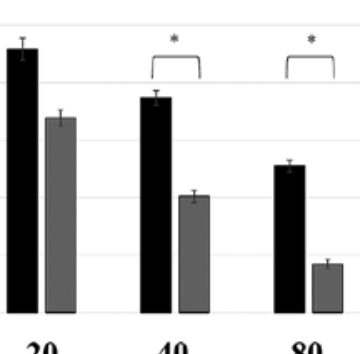

C
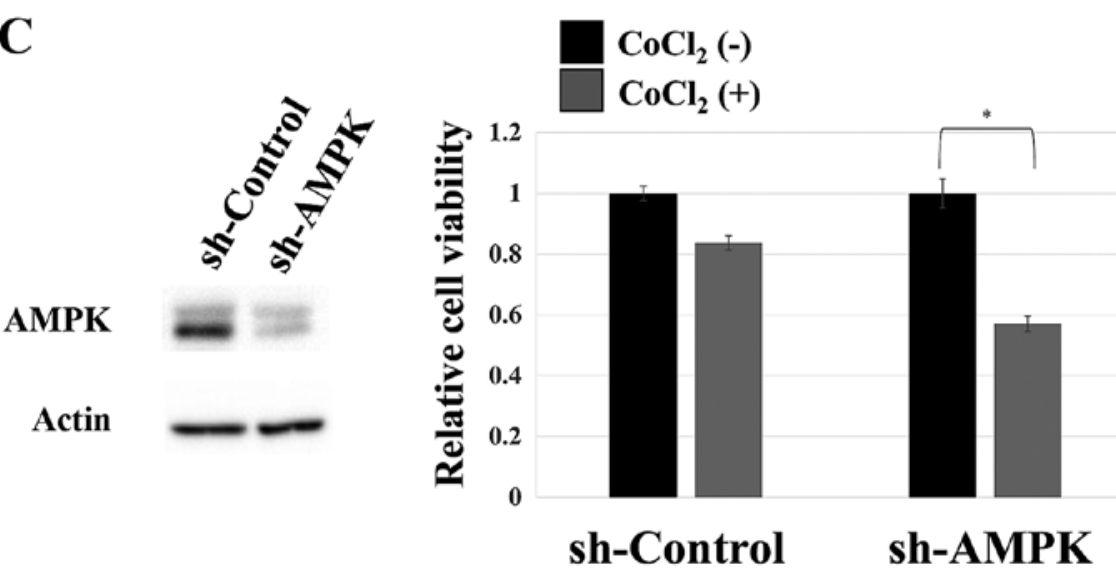

Figure 3. Significant cytotoxicity caused by autophagy inhibition under hypoxia-mimicking conditions. (A) The CCK-8 assay was used to assess the viability

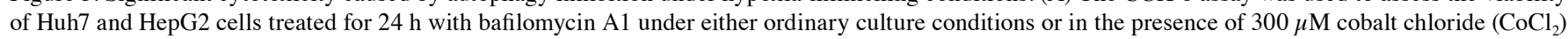
( $\mathrm{P}<0.05$ ), (B) Huh7 cells treated for $24 \mathrm{~h}$ with various concentrations of LY294002 ("P<0.05). (C) Relative cell viability following AMPK knockdown (sh-AMPK) in HepG2 cells treated for $24 \mathrm{~h}$ under the indicated conditions $(" \mathrm{P}<0.05)$. 
A
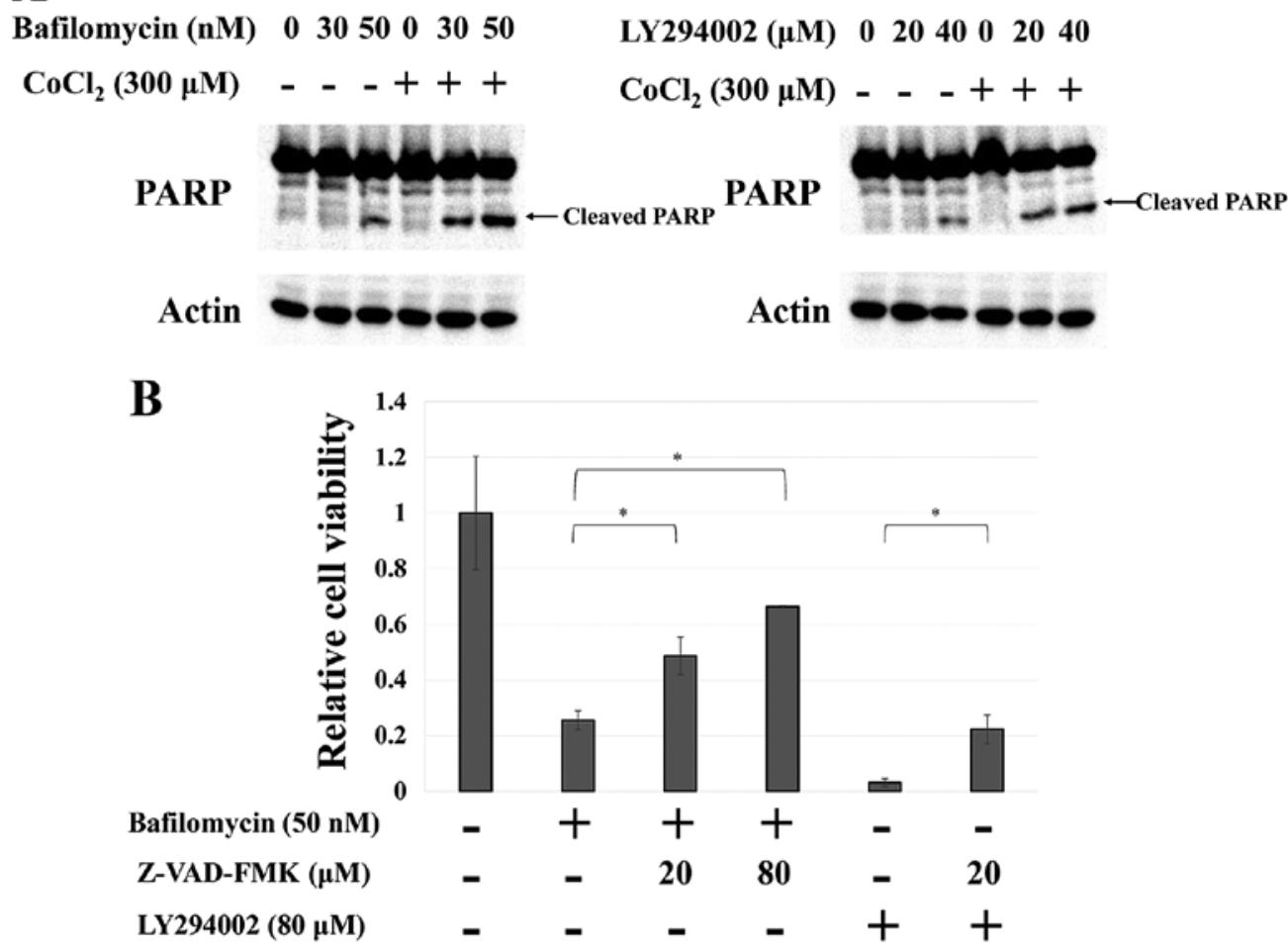

Figure 4. Autophagy induced by cobalt chloride suppresses apoptosis. (A) The expression levels of PARP and actin in Huh7 cells treated with bafilomycin A1 or LY294002 with/without $300 \mu \mathrm{M}$ cobalt chloride $\left(\mathrm{CoCl}_{2}\right)$ for $24 \mathrm{~h}$ were determined by western blot analysis. (B) The CCK-8 assay was used to assess the viability of Huh7 cells treated with bafilomycin or LY294002 with/without Z-VAD-FMK in the presence of $300 \mu \mathrm{M}$ cobalt chloride ( $\mathrm{P}<0.05$ ).

phosphorylation), based on which pathways are involved. However, as demonstrated in Fig. 2B, the phosphorylation of both AKT and AMPK was increased (Fig. 2B). Thus, we concluded that the induction of autophagy by cobalt chloride treatment is likely to be independent of the AKT pathway and dependent on the AMPK/mTOR pathway. This result was consistent with a previous study that examined the mechanism of hypoxia-induced autophagy in human dental pulp cells (30).

Autophagy inhibition results in selective cytotoxicity under hypoxia-mimicking conditions. Both hepatocellular carcinoma cell lines were treated with bafilomycin A1, an autophagy inhibitor $(31,32)$. In both cell lines, the inhibitor displayed modest toxicity under normal culture conditions, whereas it dramatically reduced cell survival when added to cells in hypoxia-mimicking conditions (Fig. 3A). High toxicity was also observed when the Huh7 cells were treated with LY294002, a different autophagy inhibitor (33), under hypoxia-mimicking conditions (Fig. 3B). As aforementioned, AMPK probably mediates the induction of autophagy by cobalt chloride treatment. Thus, we attempted to examine the role of this kinase in the adaptation of cells to this condition. As displayed in Fig. 3C, the knockdown of AMPK was extremely toxic under hypoxia-mimicking conditions in HepG2 cells. These results strongly indicated that the proper functioning of autophagy is essential for the adaptation to hypoxia-mimicking conditions and that the AMPK pathway plays a critical role in the process of adaptation as it mediates cobalt chloride-induced autophagy.
Cobalt chloride-induced autophagy suppresses apoptosis. It has been reported that autophagy supports cell survival through the suppression of apoptosis (34). Thus, we examined whether apoptosis would be induced in the Huh7 cells when adaptation to hypoxia-mimicking conditions was blocked by autophagy inhibitors. Notably, inhibiting autophagy under hypoxia-mimicking conditions resulted in a large increase in PARP cleavage (Fig. 4A), which is an apoptosis indicator, whereas co-treatment with z-VAD-FMK, an apoptosis inhibitor, strongly attenuated this effect (Fig. 4B). These results indicated that hepatocellular carcinoma cells adapted to hypoxia using autophagy to suppress apoptosis.

Mitophagy is a critical adaptive response tohypoxia-mimicking conditions. It has been reported that cells use autophagy to destroy mitochondria in order to maintain the intracellular redox status, which allows them to evade apoptosis (17). This 'mitochondria-selective' type of autophagy is termed mitophagy. We examined whether mitophagy is induced during adaptation to hypoxia-mimicking conditions by immunostaining HepG2 cells for LC3 and Tom20, a known mitochondrial marker. The distribution of most of the LC3 puncta corresponded to one of the Tom 20 puncta under hypoxia-mimicking conditions, strongly indicating that mitophagy had been induced (Fig. 5A and B). In addition, treatment with MitoTEMPO, a mitochondria-selective antioxidant reagent (35), significantly reduced cytotoxicity and mitochondrial reactive oxygen species (ROS) resulting from the inhibition of autophagy during hypoxia-mimicking conditions (Fig. 5C and D). These results indicated mitophagy as the 

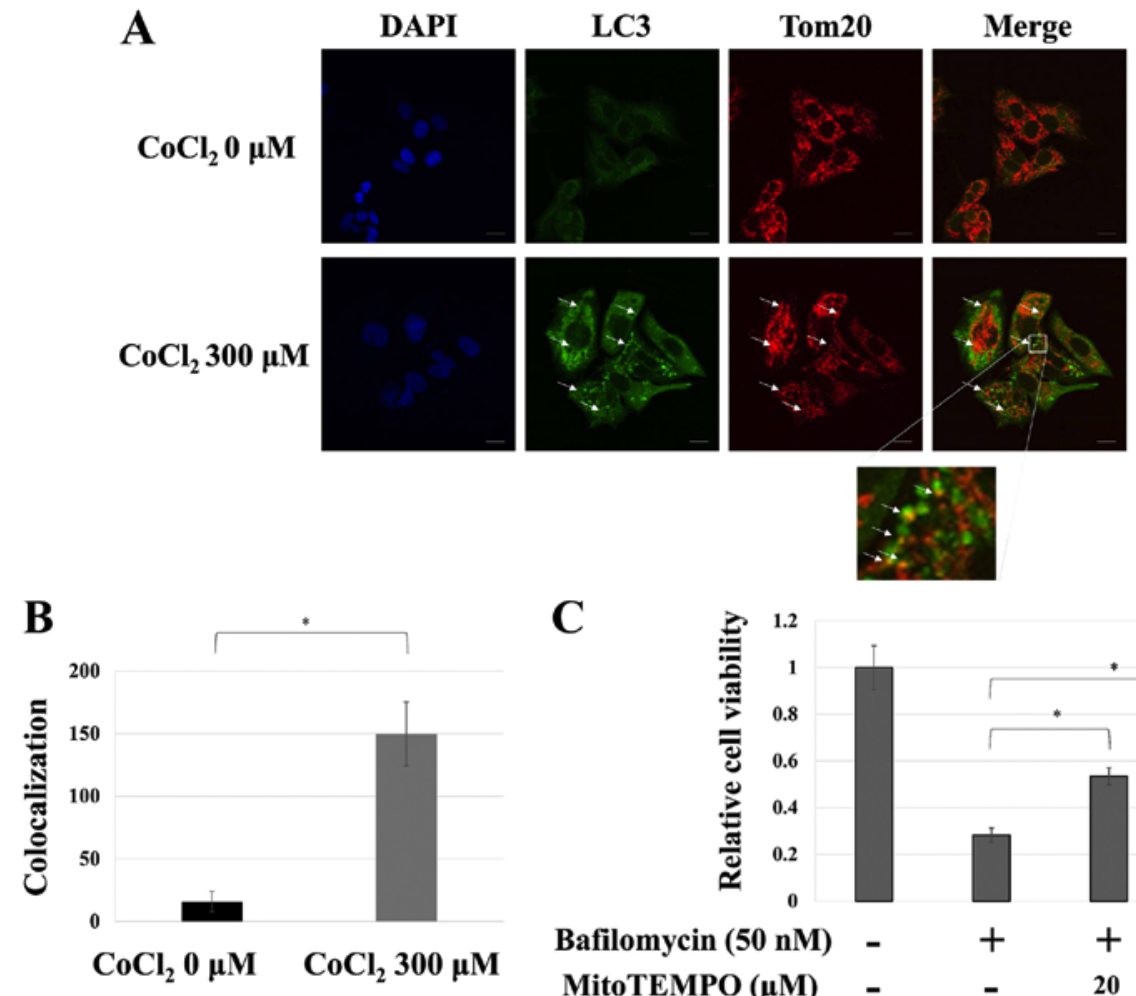

D

C
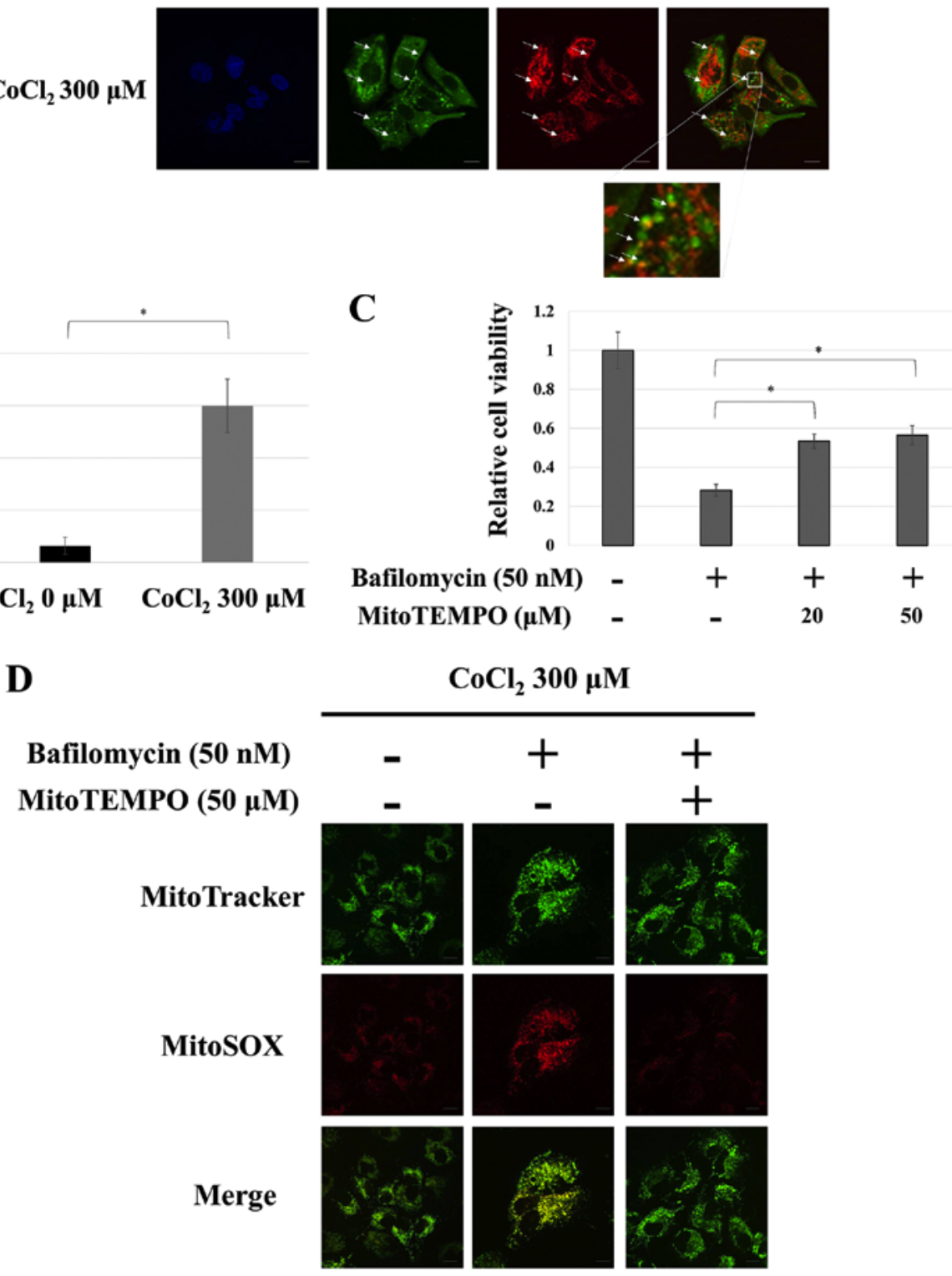

$\mathrm{CoCl}_{2} 300 \mu \mathrm{M}$

Figure 5. Mitophagy is essential to adaptation to hypoxia-mimicking conditions. (A) HepG2 cells were treated with $300 \mu \mathrm{M}$ cobalt chloride $\left(\mathrm{CoCl}_{2}\right)$ for $24 \mathrm{~h}$ and immunostained with anti-LC3 (green) and anti-Tom 20 (mitochondrial marker, Red). Insets indicate magnified images of the boxed area. Bar, $10 \mu \mathrm{m}$. Arrows indicate colocalization between LC3 and Tom20. (B) Colocalization between LC3 and Tom 20 in each cell was assessed using ImageJ software ( $\mathrm{P}<0.05)$. (C) The CCK-8 assay was used to assess the viability of Huh7 cells treated for $24 \mathrm{~h}$ with bafilomycin with/without MitoTEMPO in the presence of $300 \mu \mathrm{M}$ cobalt chloride ( $\mathrm{P}<0.05)$. (D) In addition, Huh7 cells were stained with MitoTracker Green FM (100 nM) and MitoSOX Red mitochondrial superoxide probes $(2.5 \mathrm{mM})$. Insets indicate magnified images of the boxed area. Bar, $10 \mu \mathrm{m}$.

type of autophagy mainly used by hepatocellular carcinoma cells to adapt to hypoxia conditions and avoid apoptosis.

\section{Discussion}

In the present study, we used hepatocellular carcinoma cells to examine the importance of autophagy on their ability to adapt to hypoxia-mimicking conditions, which were achieved using cobalt chloride treatment. We found that cobalt chloride induces autophagy through the AMPK pathway and that this process allows cells to evade apoptosis and adapt to the hypoxia-mimicking environment. Furthermore, autophagy inhibitors exhibited significant cytotoxicity under hypoxia-mimicking conditions. Since cobalt chloride induces stabilization of HIF-1 and is known as a hypoxia-mimicking agent, further investigation of the effects of exposure to other hypoxia-mimicking agents and to a hypoxic environment is necessary to gain a more precise understanding of the significance of autophagy in the process of adaptation to hypoxic environments. 
It is known that AMPK can be activated by two distinct mechanisms (36). The first, which is considered the classical activation mechanism, is sensing the intracellular ATP/AMP ratio. Notably, the intracellular ATP level has been reported to decrease under hypoxic conditions (37). Thus, the activation of AMPK in the present hypoxia-mimicking cell culture system may be attributed, at least partly, to this mechanism. The second AMPK activation mechanism is stimulation by ROS (36). It has recently been revealed that, beyond their ability to damage DNA and proteins, ROS also play an important role as mediator molecules controlling cellular signaling when their levels are low $(38,39)$. More important with respect to our findings, is that hypoxia is known to cause an intracellular accumulation of ROS, which are produced both in mitochondria and by the activity of NOX4 and contribute to cell proliferation, survival and motility $(40,41)$. These data indicate that the phagocytosis-inducing activation of the AMPK pathway in our hypoxia-mimicking cell culture system may be attributed to either of the two known AMPK activation mechanisms. Further studies are required to determine the extent of the contribution of each mechanism. In addition, it is important to note that the AKT phosphorylation was enhanced by the treatment with cobalt chloride in the present study. The activation of AKT is involved in cell survival and proliferation. In turn, LY294002, an autophagy inhibitor used in this study, is known to be the pan-PI3K inhibitor. Therefore, it was difficult to clearly distinguish whether the high level of cytotoxicity induced by LY294002, during cobalt-chloride treatment, was due to the inhibition of autophagy or the blockade of the survival signal of AKT. It is necessary to elucidate the detailed role of AKT in hypoxic adaptation in the future.

It has been reported that autophagy induced in a hypoxic environment negatively affects survival in glioma and breast cancer cell lines, as autophagic cell death is triggered under these conditions (21). To our knowledge, the present study is the first to report an anti-apoptotic, pro-survival effect for cobalt chloride-induced autophagy in hepatocellular carcinoma cell lines, indicating that autophagy may constitute a potential therapeutic target for this type of cancer. Furthermore, our results indicated that mitophagy is a critical system that allows cells to adapt to hypoxia through the regulation of intracellular ROS levels. This is both rational, since mitochondria serve as major loci of ROS production and consistent with previous data that support a mitophagy-mediated apoptosis avoidance mechanism (17). Thus, it is likely that cells avert excessive ROS accumulation by properly processing mitochondria damaged by hypoxic stress via mitophagy.

Notably, the present study indicated that the cytotoxicity of autophagy inhibitors was selective to hypoxia-mimicking conditions, as their effect on cell survival under normoxic culture conditions was limited. Since the reduced form of glutathione, which determines the cellular antioxidant capacity, decreases in a hypoxic environment (42), the selective character of the cytotoxicity displayed by autophagy inhibitors may be attributed to excess ROS being produced due to mitophagy failure to overwhelm the antioxidant capacity of the cell, mostly when this capacity is already compromised, such as under hypoxia-mimicking conditions. Under normoxic conditions, conversely, the small number of damaged mitochondria and the intact glutathione-based anti-oxidant defense maintains cytotoxicity at relatively low levels despite autophagy failure. Future studies focusing on the intracellular redox status may be required in order to elucidate the underlying molecular mechanism of these processes.

Considering the microenvironments that can be found inside a tumor, the dependence of cancer tissues on the bloodstream to obtain oxygen and nutrients causes parts of the tumor to be exposed not only to hypoxia, but also to a lack of nutrients. Identifying valid therapeutic targets in these microenvironments requires determining in what way cancer cells adapt to both types of stress and, importantly, discovering mechanisms that may be shared among the two adaptation processes. Since autophagy in hepatocellular carcinoma cells is induced even in a nutrient-poor environment, supporting cell survival (Endo et al unpublished data), it may represent such a common mechanism. Consistent with the importance of autophagy in tumor microenvironment, an analysis using human surgical specimens indicated that cancerous tissues had a higher autophagic activity than non-cancerous tissues (43). Therefore, autophagy may be viewed as a potential therapeutic target not only in the context of the hypoxic stress response, but also in the whole cancer microenvironment.

In the present study, we revealed that autophagy induced by cobalt chloride treatment did not trigger autophagic cell death in hepatocellular carcinoma cells but rather contributed significantly to cell survival. Additionally, we demonstrated that autophagy may constitute a very attractive target for developing novel therapeutic strategies for the treatment of hepatocellular carcinoma.

\section{Acknowledgements}

We wish to thank Makiko Sato and Akiko Sakuyama from the Department of Preventive Medicine, Tokai University School of Medicine, for their excellent secretarial support and Kazuhiro Yoshida from the Support Center for Medical Research and Education, Tokai University, for providing excellent technical support. We would also like to thank Editage (www.editage.jp) for English language editing. The present study was supported in part by the 2016 Research and Study Project of Tokai University Educational System General Research Organization (SO), the 2016 Tokai University School of Medicine Research Aid (SO), the 2017 Research and Study Program of Tokai University Educational System General Research Organization (SO), the 2017 Tokai University School of Medicine Research Aid (HE) and a Grant-in-Aid for Scientific Research (nos. 23701113 and 26870600 to HE) from the Ministry of Education, Culture, Sports, Science and Technology of Japan.

\section{Competing interests}

The authors declare that they have no competing interests.

\section{References}

1. Ferlay J, Soerjomataram I, Dikshit R, Eser S, Mathers C, Rebelo M, Parkin DM, Forman D and Bray F: Cancer incidence and mortality worldwide: Sources, methods and major patterns in GLOBOCAN 2012. Int J Cancer 136: E359-E386, 2015.

2. Llovet JM, Ricci S, Mazzaferro V, Hilgard P, Gane E, Blanc JF, de Oliveira AC, Santoro A, Raoul JL, Forner A, et al: Sorafenib in advanced hepatocellular carcinoma. N Engl J Med 359: 378-390, 2008. 
3. Wörns M and Galle PR: HCC therapies-lessons learned. Nat Rev Gastroenterol Hepatol 11: 447-452, 2014.

4. Brown JM and Giaccia AJ: The unique physiology of solid tumors: Opportunities (and problems) for cancer therapy. Cancer Res 58: 1408-1416, 1998.

5. Jain RK: Molecular regulation of vessel maturation. Nat Med 9: 685-693, 2003

6. Less JR, Skalak TC, Sevick EM and Jain RK: Microvascular architecture in a mammary carcinoma: Branching patterns and vessel dimensions. Cancer Res 51: 265-273, 1991.

7. Thomlinson RH and Gray LH: The histological structure of some human lung cancers and the possible implications for radiotherapy. Br J Cancer 9: 539-549, 1955.

8. Helmlinger G, Yuan F, Dellian M and Jain RK: Interstitial $\mathrm{pH}$ and $\mathrm{pO}_{2}$ gradients in solid tumors in vivo: High-resolution measurements reveal a lack of correlation. Nat Med 3: 177-182, 1997.

9. Vaupel P, Höckel M and Mayer A: Detection and characterization of tumor hypoxia using $\mathrm{pO}_{2}$ histography. Antioxid Redox Signal 9: 1221-1235, 2007.

10. Heindryckx F, Mertens K, Charette N, Vandeghinste B, Casteleyn C, Van Steenkiste C, Slaets D, Libbrecht L, Staelens S, Starkel P, et al: Kinetics of angiogenic changes in a new mouse model for hepatocellular carcinoma. Mol Cancer 9: 219, 2010.

11. Liu L, Ning X, Sun L, Zhang H, Shi Y, Guo C, Han S, Liu J, Sun S, Han Z, et al: Hypoxia-inducible factor-1 alpha contributes to hypoxia-induced chemoresistance in gastric cancer. Cancer Sci 99: 121-128, 2008

12. Song J, Qu Z, Guo X, Zhao Q, Zhao X, Gao L, Sun K, Shen F, Wu M and Wei L: Hypoxia-induced autophagy contributes to the chemoresistance of hepatocellular carcinoma cells. Autophagy 5 1131-1144, 2009.

13. Sullivan R,ParéGC,Frederiksen LJ,Semenza GLand Graham CH: Hypoxia-induced resistance to anticancer drugs is associated with decreased senescence and requires hypoxia-inducible factor-1 activity. Mol Cancer Ther 7: 1961-1973, 2008.

14. Yokoi K and Fidler IJ: Hypoxia increases resistance of human pancreatic cancer cells to apoptosis induced by gemcitabine. Clin Cancer Res 10: 2299-2306, 2004.

15. Semenza GL: Hypoxia-inducible factor 1: Master regulator of $\mathrm{O}_{2}$ homeostasis. Curr Opin Genet Dev 8: 588-594, 1998.

16. Semenza GL: HIF-1 and tumor progression: Pathophysiology and therapeutics. Trends Mol Med 8: S62-S67, 2002.

17. Zhang H, Bosch-Marce M, Shimoda LA, Tan YS, Baek JH, Wesley JB, Gonzalez FJ and Semenza GL: Mitochondrial autophagy is an HIF-1-dependent adaptive metabolic response to hypoxia. J Biol Chem 283: 10892-10903, 2008.

18. Mizushima N, Yamamoto A, Matsui M, Yoshimori T and Ohsumi Y: In vivo analysis of autophagy in response to nutrient starvation using transgenic mice expressing a fluorescent autophagosome marker. Mol Biol Cell 15: 1101-1111, 2004

19. Lum JJ, Bauer DE, Kong M, Harris MH, Li C, Lindsten T and Thompson CB: Growth factor regulation of autophagy and cell survival in the absence of apoptosis. Cell 120: 237-248, 2005.

20. Bursch W, Ellinger A, Kienzl H, Török L, Pandey S, Sikorska M, Walker R and Hermann RS: Active cell death induced by the anti-estrogens tamoxifen and ICI 164384 in human mammary carcinoma cells (MCF-7) in culture: The role of autophagy. Carcinogenesis 17: 1595-1607, 1996.

21. Azad MB, Chen Y, Henson ES, Cizeau J, McMillan-Ward E, Israels SJ and Gibson SB: Hypoxia induces autophagic cell death in apoptosis-competent cells through a mechanism involving BNIP3. Autophagy 4: 195-204, 2008

22. Tasdemir E,Maiuri MC,Galluzzi L,Vitale I,Djavaheri-Mergny M, D'Amelio M, Criollo A, Morselli E, Zhu C, Harper F, et al: Regulation of autophagy by cytoplasmic p53. Nat Cell Biol 10: 676-687, 2008

23. Zhang R, Zhu F, Ren J, Huang L, Liu P and Wu G: Beclin1/PI3K-mediated autophagy prevents hypoxia-induced apoptosis in EAhy926 cell line. Cancer Biother Radiopharm 26: 335-343, 2011

24. Endo H, Niioka M, Kobayashi N, Tanaka M and Watanabe T: Butyrate-producing probiotics reduce nonalcoholic fatty liver disease progression in rats: New insight into the probiotics for the gut-liver axis. PLoS One 8: e63388, 2013.
25. Fan RH, Chen PS, Zhao D and Zhang WD: Hypoxia induced by $\mathrm{CoCl} 2$ influencing the expression and the activity of matrix metalloproteinase-2 in rat hepatic stellate cells. Zhonghua Gan Zang Bing Za Zhi 15: 654-657, 2007 (In Chinese).

26. Karovic O, Tonazzini I, Rebola N, Edström E, Lövdahl C, Fredholm BB and Daré E: Toxic effects of cobalt in primary cultures of mouse astrocytes. Similarities with hypoxia and role of HIF-1alpha. Biochem Pharmacol 73: 694-708, 2007.

27. Blommaart EF, Luiken JJ, Blommaart PJ, van Woerkom GM and Meijer AJ: Phosphorylation of ribosomal protein S6 is inhibitory for autophagy in isolated rat hepatocytes. J Biol Chem 270: 2320-2326, 1995.

28. Hahn-Windgassen A, Nogueira V, Chen CC, Skeen JE, Sonenberg N and Hay N: Akt activates the mammalian target of rapamycin by regulating cellular ATP level and AMPK activity. J Biol Chem 280: 32081-32089, 2005.

29. Inoki K, Zhu T and Guan K: TSC2 mediates cellular energy response to control cell growth and survival. Cell 115: 577-590, 2003.

30. Zhou Q, Liu H, Sun Q, Zhang L, Lin H, Yuan G, Zhang L and Chen Z: Adenosine monophosphate-activated protein kinase/mammalian target of rapamycin-dependent autophagy protects human dental pulp cells against hypoxia. J Endod 39: 768-773, 2013.

31. Bowman EJ, Siebers A and Altendorf K: Bafilomycins: A class of inhibitors of membrane ATPases from microorganisms, animal cells, and plant cells. Proc Natl Acad Sci USA 85: 7972-7976, 1988.

32. Yoshimori T, Yamamoto A, Moriyama Y, Futai M and Tashiro Y: Bafilomycin A1, a specific inhibitor of vacuolar-type $\mathrm{H}(+)$-ATPase, inhibits acidification and protein degradation in lysosomes of cultured cells. J Biol Chem 266: 17707-17712, 1991.

33. Blommaart EF, Krause U, Schellens JP, Vreeling-Sindelárová H and Meijer AJ: The phosphatidylinositol 3-kinase inhibitors wortmannin and LY294002 inhibit autophagy in isolated rat hepatocytes. Eur J Biochem 243: 240-246, 1997.

34. Kim SE, Park HJ, Jeong HK, Kim MJ, Kim M, Bae ON and Baek SH: Autophagy sustains the survival of human pancreatic cancer PANC-1 cells under extreme nutrient deprivation conditions. Biochem Biophys Res Commun 463: 205-210, 2015.

35. Murphy MP and Smith RA: Targeting antioxidants to mitochondria by conjugation to lipophilic cations. Annu Rev Pharmacol Toxicol 47: 629-656, 2007.

36. Hardie DG, Ross FA and Hawley SA: AMPK: A nutrient and energy sensor that maintains energy homeostasis. Nat Rev Mol Cell Biology 13: 251-262, 2012.

37. Heerlein K, Schulze A, Hotz L, Bärtsch P and Mairbäurl H: Hypoxia decreases cellular ATP demand and inhibits mitochondrial respiration of a 549 cells. Am J Respir Cell Mol Biol 32: 44-51, 2005.

38. Owada S, Shimoda Y, Tsuchihara K and Esumi H: Critical role of $\mathrm{H}_{2} \mathrm{O}_{2}$ generated by NOX4 during cellular response under glucose deprivation. PLoS One 8: e56628, 2013.

39. Nogueira V and Hay N: Molecular pathways: Reactive oxygen species homeostasis in cancer cells and implications for cancer therapy. Clin Cancer Res 19: 4309-4314, 2013.

40. Chandel NS, McClintock DS, Feliciano CE, Wood TM, Melendez JA, Rodriguez AM and Schumacker PT: Reactive oxygen species generated at mitochondrial complex III stabilize hypoxia-inducible factor-1alpha during hypoxia: A mechanism of $\mathrm{O}_{2}$ sensing. J Biol Chem 275: 25130-25138, 2000.

41. Diebold I, Petry A, Hess J and Görlach A: The NADPH oxidase subunit NOX4 is a new target gene of the hypoxia-inducible factor-1. Mol Biol Cell 21: 2087-2096, 2010.

42. Mansfield KD, Simon MC and Keith B: Hypoxic reduction in cellular glutathione levels requires mitochondrial reactive oxygen species. J Appl Physiol (1985) 97: 1358-1366, 2004.

43. Hirayama A, Kami K, Sugimoto M, Sugawara M, Toki N, Onozuka H, Kinoshita T, Saito N, Ochiai A, Tomita M, et al: Quantitative metabolome profiling of colon and stomach cancer microenvironment by capillary electrophoresis time-of-flight mass spectrometry. Cancer Res 69: 4918-4925, 2009. 